\section{Secondary Use and Analysis of Big Data Collected for Patient Care}

\section{Contribution from the IMIA Working Group on Data Mining and Big Data Analytics}

\author{
F. J. Martin-Sanchez ${ }^{1}$, V. Aguiar-Pulido ${ }^{2}$, G. H. Lopez-Campos ${ }^{3}$, N. Peek ${ }^{4}$, L. Sacchi ${ }^{5}$ \\ 1 Weill Cornell Medicine, Department of Healthcare Policy and Research, Division of Health \\ Informatics, New York, USA \\ 2 Weill Cornell Medicine, Brain and Mind Research Institute, New York, USA \\ ${ }^{3}$ The University of Melbourne, Health \& Biomedical Informatics Centre, Melbourne, Australia \\ ${ }^{4}$ MRC Health e-Research Centre, Division of Informatics, Imaging and Data Science, The University \\ of Manchester, Manchester, UK \\ ${ }^{5}$ Department of Electrical, Computer and Biomedical Engineering, University of Pavia, Pavia, Italy
}

and Big Data analytics" focuses on the literature published during the last two years, covering the timeframe since the working group's last survey.

Results and Conclusions: Although most of the examples of secondary use of patient data lie in the arena of clinical and health services research, we have started to witness other important applications, particularly in the area of genomic research and the study of health effects of environmental factors. Further research is needed to characterize the economic impact of secondary use across the broad spectrum of translational research.

\section{Keywords}

Electronic health record; medical informatics; data mining; clinical study; translational medical research

Yearb Med Inform 2017:28-37

http://dx.doi.org/10.15265/IY-2017-008

Published online May 8, 2017

\section{Introduction}

With the advent of technology, a wealth of data sources is becoming available, both for clinicians and biomedical researchers. Although this article presents major examples of secondary use of data extracted from sources in clinical settings, the main challenge for biomedical informatics is to allow for an effective and reliable integration of distributed, complex, and heterogeneous data sources.

Within this context, it is worth highlighting at least three scenarios that involve data integration. The first one is related to the need of permeating the barriers between clinical care, clinical research, and population health [1]. Current information systems have not been designed for cutting across these settings neither the security and confidentiality requirements, nor the appropriate levels of data aggregation, although this responds to different user needs, The second scenario involves a growing diversity in data sources, derived from the availability of different technologies and origins (e.g., genetic, molecular, clinical, environmental, administrative, behavioral, socio-demographic). Each of these data types, as well as the expertise required to process them, poses significant challenges from a data integration perspective [2]. Finally, the third scenario refers to the stakeholder generating the data. We have transitioned from a provider-centered model to a patient-centered one [3]. Patient(person-) generated health data brings a new avenue for biomedical informatics research.

Only if these integration steps are adequately addressed, Big Data analytic approaches will yield actionable knowledge for medical purposes. The following sections will thus provide insight into successful experiences of the previous.

\section{Methodological and Technical Issues Related to the Secondary Use of Patient Data}

\section{2a Models for Data Integration}

The use of practice-based data to inform research has recently been identified as one of the main pillars for enabling precision medicine in the Learning Health System Cycle [4]. Informatics is the propellant for this process, as it provides methodologies to integrate, store, and share data from different sources, and to analyze such data for research purposes. Several efforts have recently been proposed, both in the US and in Europe, 
towards this goal. All these efforts have in common the definition of a model for data integration and of a framework for querying the data, maintaining privacy and security, and complying with ethical requirements [5].

Examples of such systems are clinical research networks (CRNs), which aggregate data coming from different sources represented through a common data model (CDM), and give users the possibility to query the data and perform distributed analytics on it. Through CDMs, it is possible to manage the variety of the sources that make up CRNs, by harmonizing variables and data types found in local repositories, with the ultimate goal of potentiating the complex distributed analytic functionalities that will take place on the data, allowing them to take advantage of larger samples stored in a common format. Examples of CDMs that have been used to develop several research infrastructures are the FDA Mini-Sentinel CDM [6], the Observational Medical Outcomes Partnership (OMOP) common data model [7], and the Informatics for Integrating Biology and the Bedside (i2b2) framework [8].

Among CRNs, PCORnet (http://pcornet. org/) is a National Patient-Centered Clinical research network born in 2014 from an initiative of the Patient-Centered Outcome Research Institute (PCORI) for community-based observational and interventional studies [9]. One of the main features of PCORnet is the direct involvement of patients in the initiative, with the inclusion of 20 Patient-Powered Research Networks (PPRNs) focusing on single health conditions and including rare diseases. Besides PPRNs, PCORnet includes 13 Clinical Data Research Networks (CDRNs), each collecting longitudinal data of at least one million patients each. Data is organized according to the PCORnet common data model [10]. The PCORnet CDM is based on the FDA Mini-Sentinel CDM, with the idea of leveraging the large set of analytic tools and expertise developed for that system, including data characterization approaches, and tools for complex distributed analytics. The CDM, now at its fourth release, aggregates information according to several domains that depend on the source data comes from, such as data captured from healthcare delivery and patient-reported outcomes.
Within PCORnet, health centers belonging to the Scalable Collaborative Infrastructure for a Learning Health System (SCILHS) $\mathrm{CRN}$ are all using the i2b2 data warehouse to store and analyze patient data for research. To avoid duplicating extraction-transformation-loading efforts already performed to transfer data from the hospital databases to the $\mathrm{i} 2 \mathrm{~b} 2$ data warehouse, the $\mathrm{i} 2 \mathrm{~b} 2$ project has defined a way to translate the $\mathrm{i} 2 \mathrm{~b} 2$ data model (i2b2 ontology) directly into the PCORnet CDM [11]. The i2b2 data warehouse was successfully mapped to the PCORnet CDM database for all the sites participating to the test, demonstrating that it was feasible to enable the interchange of data between different data models.

Another interesting example of collaborative platform for data re-use is the Observational Health Data Sciences and Informatics (OHDSI) [12]. Differently from PCORnet, OHDSI is mainly focused on data analytics solutions to be applied to networks of health databases [13]. OHDSI is based on the Observational Medical Outcomes Partnership (OMOP) common data model [7], and provides a set of tools for data visualization, summary statistics, and analysis.

\section{2b Omics and Big Data}

Next generation sequencing technologies are evolving at a very fast pace, outpacing Moore's Law. These advances together with the decrease in sequencing costs have resulted in a deluge of biological data being generated [14]. As a consequence, computational challenges that have to do not only with data storage but also with data processing and analysis have arisen. Furthermore, data processing is currently the most challenging of the three and has become an evident bottleneck.

For now, we can say that Big Data technologies are still in their early stages. Although powerful, there is clearly room for improvement [15]. Regarding clinical data storage, data warehouses are widely employed as a means to integrate data from different clinical sources. Clinical data warehouses (CDW), thus, provide a unified view on clinical data. Many implementations of these warehouses rely on the i2b2 infrastructure and must be appropriately adapted to handle molecular biology data, as well as to enable clinical and "omics" data integration. As part of the solutions that have emerged to deal with data management and analysis, translational research platforms seem to be on the rise. Canuel and colleagues analyzed seven of the main non-commercial translational research platforms (BRISK [16], caTRIP [17], cBio Cancer Genomics Portal for Cancer Genomics [18], Georgetown Database of Cancer - G-DOC - [19], integrated clinical omics database - iCOD - [20], integrating data for analysis, anonymization and sharing - iDASH - [21] and tranSMART [22]) in their review [23]. Most of these platforms rely heavily on third-party widely adopted solutions for core components which deal with data storage (e.g., the i2b2 CDW model) or analysis (e.g., the R statistical framework).

Although these platforms represent a first step in the development of tools and methods that enable exploring and managing very diverse data, more advances will be required to fully take advantage of the available data. One key issue identified by the previous authors has to do with privacy. In this sense, several platforms feature a client/server architecture, for which the server is not controlled by the final user. Given the nature of the data, this could involve data privacy issues under the existing regulations. Furthermore, installing the platform in the institution that "owns" the data could be mandatory in many situations. Another major caveat pinpointed by the authors is the fact that none of the reviewed platforms directly support international exchange standards such as HL7 CDA or CDISC ODM. As a result, none of them will be able to import data from EHRs or personal health records. Furthermore, these platforms only present limited capabilities regarding the use of standard terminologies and ontologies, restricting interoperability and data sharing. Finally, the management of complex temporal data has yet to be solved.

Parallelized pipelines will need to be created in order to process "omics" data in a timely manner. Currently, few bioinformatics tools offer parallelization given the complexity involved in their design; some efforts in this arena are discussed by O'Driscoll and colleagues [15]. Cloud computing has also 
become very popular recently and could be applied to "omics". However, in order to take full advantage of the capabilities offered by the cloud, and given the scale of the data involved, new solutions have to be provided regarding data transfer. Uploading and downloading "omics" data from/to the cloud becomes a bottleneck, since the transmission rate is limited. Again, issues with the privacy of medical and "omics" data will have to be tackled. In clinical settings, strong regulations, such as the Health Insurance Portability and Accounting Act of 1996 (HIPAA), will apply.

Lastly, with the launch of precision medicine, new approaches will have to be devised in order to integrate phenotypic, genotypic, and environment-related data. The previously mentioned $12 \mathrm{~b} 2$ system provides a useful framework which can be successfully applied to this type of approach [24]. Based on this framework, approaches such as BigQ have been developed to deal with genomic variants in a clinical research environment [25].

\section{2c Main Advantages}

The main advantages of re-using routinely-collected data for research is that such studies can be conducted on a larger scale, against lower costs, and within shorter time frames than traditional studies [26]. In particular, such studies can have very large cohort sizes and long follow-up times, thus enabling reliable estimation of rare outcome incident rates and long-term effects of interventions. The cost of conducting these studies is much lower than for conventional studies because no staff is needed to implement study protocols and record data. So, it is mostly the 'Volume' aspect of Big Data that is attractive here. Moreover, the real-world setting in which the data is collected increases the external validity of such studies compared to more traditional designs such as controlled trials. For instance, there will be little or no Hawthorne effects - the phenomenon that people tend behave differently when they know that they are being observed.

We illustrate some of these advantages with two recent studies. Matthews and co-workers performed a population-based study of diagnostic medical radiation exposure [27] - a field that had so far largely relied on the information produced by a single study in Japanese atomic bomb survivors. They assessed the cancer risk following exposure to low dose of ionizing radiation from diagnostic computed tomography (CT) scanning in a cohort of 10.9 million individuals in Australia by linking electronic patient records from the federally funded Australian Medicare system to the Australian Cancer Database and the National Death Index. The mean follow-up time was 9.5 years for the group exposed to CT scanning and 17.3 years for the unexposed group. They found that cancer incidence was $24 \%$ greater in people exposed to CT scanning, after accounting for age, sex, and year of birth using Poisson regression analysis. This finding allows practitioners to better weigh the diagnostic benefits of CT scans in clinical practice against their adverse effects.

A roughly similar design allowed Been et al. [28] to evaluate the effects of smoke-free legislation, a population-level public health intervention, on health outcomes. During the 2000s, many Western countries introduced legislation to prohibit smoking in enclosed public places and the workplace. Yet, while there was ample evidence that tobacco smoking is the primary cause of preventable mortality worldwide, little was known about the actual health benefits of such smoking bans. The study assessed the impact of UK smoke-free legislation, introduced in July 2007, on perinatal survival by linking individual-level data with death certificates for all registered singleton births in England over the time period 1995-2011, to obtain a dataset of 52 thousand stillbirths and 10.2 million livebirths. Using interrupted time series logistic regression analysis and counterfactual reasoning, it was estimated that in the first four years after the smoking ban, 991 stillbirths and 430 neonatal deaths were prevented.

\section{2d Data Quality}

Notwithstanding the benefits of re-using routinely collected data for research, there are also significant risks of bias due to the nature and quality of data. Routinely acquired data differs from data collected primarily for the purposes of research: we cannot assume that such data provides a full or accurate clinical picture, let alone a full description of the health of the population under study [29]. With data recorded for administrative purposes (reimbursement of costs) or direct care (patient records), recording is typically driven by the purpose of the clinical encounter that is described by the data, and other things will not be recorded. But absence of recording a disease does not mean absence of the disease. If a patient's blood pressure is not measured, this doesn't mean that the patient does not have hypertension. Similarly, mild pain is often treated using over-the-counter medications leaving no mark in the medical record [30, 31].

This situation creates significant challenges for cohort selection, assessment of co-morbidities and confounders, and assessment of health outcomes. Researchers have therefore warned that epidemiological studies with routinely collected health data, including the evaluation of interventions, is potentially subject to multiple sources of bias [32]. For instance, many studies limit the included cohort to patients for whom there are at least two sets of laboratory results (e.g., two subsequent creatinine measurements to assess kidney function). However, Rusanov and colleagues showed that this type of selection is biased towards sick patients [31].

Further data quality issues relate to variation in coding practice [33], bias in recording due to financial incentives, and the use of unstructured data such as free text.

\section{Review of Recent Studies on the Secondary Use of Patient Data}

\section{3a Secondary Use of Patient Data for Clinical Research}

The widespread availability of data collected at different steps of the clinical management of patients (clinical routine, administrative flow, insurance claims) has opened several perspectives for reusing such data in clinical research and, subsequently, in patient care [34]. In this section, we review some 
of the most recent literature in the area, particularly referring to works published in 2015 and 2016.

Since the first experiences in data re-use $[35,36]$, attention has been focused on the selection and the definition of cohorts of patients to be used in clinical trials. This application well represents the potential intersections between clinical research and patient care, as several data elements useful for clinical trials execution are often already available in electronic health records (EHRs), and their re-use could lead to faster recruitment and decreasing costs, by reducing redundant data capture. Starting from this need, EHR4CR [37], an Innovative Medicine Initiative (IMI) funded project, was aimed at building a platform to support clinical trials based on routinely collected data from EHR systems in Europe [38]. Besides clinical trials execution, the reporting of serious adverse events is also addressed by the EHR4CR project [39]. The EHR4CR network, which included 11 hospitals across Europe, was designed using an architecture promoting the re-use of already available solutions and connect single-center EHRs to the platform. Semantic mapping between local terminologies and the central EHR4CR terminology led to the definition of the EHR4CR Common Information Model. The EHR4CR project ended in 2015 and the platform is currently being scaled up across Europe. Therefore we expect that analyses of data coming from the platform will start being presented. Up to now, a cost benefit assessment analysis of using EHR data versus current practices has been proposed, showing that the EHR4CR platform would enable a $50 \%$ reduction of person-time and operational costs when conducting clinical research scenarios in an oncology clinical trial [40].

Another ongoing EU-funded initiative on data re-use for research is the European Medical Information Framework (EMIF) [41]. This project is focused on the development of a platform able to aggregate data coming not only from clinical EHRs, but also from other sources, such as already performed cohort studies, registries, administrative data, genomic data, and biobanks. The project is mainly focused on identifying predictors for metabolic complications in obesity and for Alzheimer's disease, and has already succeeded in creating large sample sets for biomarker discovery by merging patients cohorts spread across Europe using an OMOP-based common data model [42].

In the US, some important projects related to data re-use are also present. One example is the Shared Health Research Information Network (SHRINE) [43], developed at Harvard Medical School as one of the first networks that allowed researchers to perform federated queries on populations of patients aggregated across many hospitals. In analogy with $i 2 b 2$, which is the default platform SHRINE is designed to work on, SHRINE common data model is based on an ontology (called the Core Ontology) that defines the set of medical concepts that can be used to query the system. On the commercial side, a successful example is represented by the Trinetx network (http://trinetx.com/), a cloud-based health research platform that allows healthcare organizations, pharmaceutical companies, and research organizations to collaborate in the process of trial design and patient recruitment.

The automatic selection of patients eligible for a clinical trial is one of the applications of what is generally known as electronic phenotyping [44], a concept that is strictly linked to the reuse of patient data for research and that refers to a series of methodologies to derive relevant features from EHR data and use such features to answer relevant research questions [45]. Beyond supporting the recruitment of patients for clinical trials, electronic phenotyping can be used for the stratification of patient cohorts and for the implementation of decision support tools to be embedded within EHR systems [45]. Several advanced and robust methodologies have been proposed to extract phenotypes from routinely collected clinical data and reuse the results for research [46]. As part of the $\mathrm{i} 2 \mathrm{~b} 2$ project, one general approach was defined that allows developing a robust phenotype algorithm to be applied to several clinical domains, like diabetes, depression, multiple sclerosis, and rheumatoid arthritis [47]. Such algorithm, besides considering the structured data included in EHRs, also uses natural language processing (NLP) to extract information from clinical text reports and notes. Once relevant domain-specific features are defined for the domain of interest and mapped to standard terminologies, they are extracted from structured and unstructured data and ranked using a feature selection technique. A logistic regression classification algorithm is finally used to assign to each patient a probability of having the desired phenotype.

To overcome the limitations of manual curation of the initial set of features that defines a phenotype, automatic algorithms have been proposed. In [48], features are selected using publicly available knowledge sources and NLP. The recently proposed "deep patient" framework uses unsupervised feature learning to extract a set of general, domain-independent, features from EHR databases [49]. Deep patient exploits a deep learning approach based on multi-layer neural networks to extract a compact, high-level, representation of the patient. The features extracted using this methodology are used to predict patients' future diseases.

Time has also been identified as a relevant feature that is naturally embedded by the data collection process in clinical practice. Taking into account the dynamics of the collected data allows specifying a novel way to stratify the population, called dynamical phenotyping [50]. Besides clustering patients based on their dynamics [51], novel techniques have been proposed to mitigate some of the biases that characterize data collected in clinical routine, namely irregular sampling time and non-stationarity [52], and to preserve temporal relationships in a privacy-safe context [53].

Besides integrating data and making query services available through users, systems like i2b2 have been recently enhanced with some advanced analytics functionalities that go beyond the original goal of cohort selection. An example is given by the functionalities for statistical analysis implemented in the Analytics cell for $\mathrm{i} 2 \mathrm{~b} 2$ [54]. The applications currently included in this framework allow performing summary statistics on a patient set, testing hypotheses with suitable data visualization, and the possibility to draw survival curves.

While data reuse for research has been broadly addressed in the past two years, its effective adoption in patient care is less common. Although the majority of 
the systems based on clinical data reuse are expected to have an impact on patient care in the future, the number of systems that are already adopted in clinical practice is still very limited. This is due to the fact that closing the learning healthcare system cycle by transferring the results of research to the clinical practice is a complex process. Nevertheless, some examples of systems that already use available clinical data to improve decision support tools already exist. One example is given by a web-based population risk surveillance dashboard developed to predict and visualize the risk of future admissions to the emergency departments relying on data coming from EHRs and Health Information Exchange (HIE) [55]. The system is developed as a web application that integrates a predictive model validated on a large cohort of patients that provides real-time visualization of the population risk profile. The system is running on the Maine State Health Information Exchange system. Another example of a system based on the re-use of clinical data to support patient care is described in [56]. This system is aimed at supporting clinicians and healthcare managers in the management of type 2 diabetes and prevention of complications. This system is designed as a dashboard including several analytics algorithms working both at the population and the single-patient level. The developed models are derived thanks to the re-use of routinely collected clinical and administrative information, which are integrated exploiting the $\mathrm{i} 2 \mathrm{~b} 2$ technology.

\section{3b Secondary Use of Patient Data for Genomic Research and to Study the Effect of the Environment on Health}

Besides secondary use of patient data for clinical research and patient care, there are other applications, most of which are at the intersection of EHRs and genetics. In this context, biorepositories play a crucial role in generating data for potential secondary use. However, in general, the data available for this type of secondary use is very heterogeneous. Although several efforts are being made regarding data integration and standardization, there exist many private biorepositories scattered around the globe, which in many cases may have been created as a result of studies initiated by different groups of investigators. Most of this data has not been carefully organized for future reuse. As mentioned earlier, integrative approaches have to be developed to overcome the current landscape. In this section, we will review the most recent (2015-2016) and relevant works that deal with secondary use of patient data in the field of genomics and regarding the effect of the environment on human health.

With the decrease in cost of technology, it is now possible to perform multi-omics high-throughput sequencing at a reasonable price and time. However, in the case of whole genome sequencing, for example, the interpretation of the extremely high number of variants obtained both in coding and non-coding regions represents a computational challenge that has yet to be fully solved. Dried blood spots (DBSs) have been collected on a regular basis from newborns in many countries for screening purposes. Although not free of controversy (especially in the US), these collections are becoming a wealth of biological data for research. According to a study carried out in the Netherlands, Dutch mothers lean towards supporting secondary use of this data. However, more efforts are required in order to involve parents in developing policies that deal with DBSs [57]. Several researchers have used DBS data to gain more insight into different conditions and disorders. DBSs have been even used to study how the effect of the environment can affect several generations as demonstrated by Sen and colleagues in their work on inheritance of epigenetic changes associated with exposure to lead [58].

Other examples of secondary use of routine genetic data involve the monitoring of HIV transmission hotspots [59], and the prediction of long-term risk of severe infection using data from three different population-based studies, namely the Dietary, Lifestyle, and Genetic Determinants of Obesity and Metabolic syndrome (DILGOM) Study, the FINRISK Study, and the Cardiovascular Risk in Young Finns Study (YFS) [60]. In the former, researchers built a monitoring system around a cached database query, which contains de-identified data for all individuals enrolled in the British Colum- bia drug treatment program. Data is clustered based on phylogenetic distance and clusters are then annotated with clinical and demographic information. Finally, the resulting analysis is presented to the user through a graphical interface as a network. In the latter, network analysis is used to obtain modules of co-expressed genes which are then annotated with Gene Ontology (GO) and KEGG to look for enrichment. Regression models are also used to analyze the rest of the data.

The Electronic Medical Records and Genomics network (eMERGE) is a remarkable initiative organized and funded by the National Human Genome Research Institute (NHGRI). eMERGE was created to evaluate the possibility of using data from routine clinical care retrieved from electronic medical records (EMRs) to characterize disease phenotypes in genome wide association studies (GWAS) [61]. Within this initiative, a variety of methods and best practices were developed for utilizing EHRs as a tool for genomic research $[62,63]$. Before widely exploiting this data for research, several authors worked to prove the benefits of EHR-derived phenotyping. Thus, Verma and colleagues used EMR data from this network to validate the utility of EMRs to detect non-spurious and relevant associations in glaucoma [64]. For this purpose, authors carried out a GWAS using logistic regression models. After that, gene-gene interaction and pathway analyses were performed, replicating the study on the NEIGHBOR consortium dataset. EHR phenotyping has been further validated in other contexts, such as the study of liver function [65].

An electronic phenotyping algorithm was developed within the eMERGE network. This algorithm allowed Almoguera and colleagues to obtain more comprehensive information on asthma [66]. Furthermore, electronic phenotyping enabled these researchers to obtain a more accurate and objective definition of the condition, basing its diagnosis on ICD9 codes and the prescription of specific medication. As a result, the authors were able to unravel pathways that could help in improving the treatment of underlying causes of asthma. In addition to the algorithms devised as part of the eMERGE initiative, other methods have arisen to complement traditional 
genotype-phenotype studies also deriving phenotypes from EMRs, such as those developed and applied in the Epidemiologic Architecture for Genes Linked to Environment (EAGLE) study $[67,68]$.

The possibility of conducting several GWAS at the same time with no additional cost (especially economic) is very beneficial. The previous studies prove that EHRs provide a new avenue for reusing already existing genotypes by studying their relationships with different phenotypes. In addition to disease and trait genetics, EHRs are also a precious resource for investigating pharmacogenetic traits [69] and developing reverse genetics approaches such as phenome-wide association studies (PheWAS) [70]. PheWAS allow generating hypotheses by testing a selected group of single nucleotide polymorphisms (SNPs) and many phenotypes. Therefore, in the past few years, with the proliferation of data available for secondary use, these studies have been on the rise. A comprehensive review of the use of EHRs in this type of study has been done by Bush and colleagues [71]. Finally, Denny and colleagues underscored the relevance of PheWAS in the context of precision medicine, identifying this type of approach as key to new discoveries [70].

Although relatively little attention has been given to the extraction of data regarding determinants of health from EMRs, this is an area with many opportunities that will flourish in the near future. This type of data will help improve not only healthcare of individual patients, but will also enable population-level applications [72]. For this purpose, social and medical data will need to be integrated and aggregated, leading to major changes in healthcare, improving value-based payment, and eventually reflecting on the advancement of population health.

\section{3c Secondary Use of Patient Data for Evaluation of Health Interventions}

An increasing number of studies re-use routinely collected health data to evaluate the effects of health interventions. Typically, these studies retrospectively extract baseline and follow-up data from individuals that were exposed to the intervention, and from a similar group of individuals not exposed serving as controls. Often, data from different sources are linked to ensure that health outcomes are reliably captured. Regression methods are used to estimate the causal effects of the interventions on health.

A key prerequisite for this type of research is that there is sufficient variation in real-world practice to evaluate interventions. If, in routine practice, everyone with a certain condition receives intervention $\mathrm{X}$, then there is no opportunity to evaluate the effects of $\mathrm{X}$ with routine data, simply because no comparison can be made. But once there is variation in practice, i.e. some patients receive the intervention while similar others do not, this offers the opportunity to view the real world as a natural experiment and draw inferences from that. So, for the evaluation of interventions, it is the 'Variation' aspect of Big Data that is essential.

As described earlier, there are many advantages to re-using routinely collected health data for research. This type of research has lower costs, larger sample sizes, longer follow-up times, and is more representative of real-world clinical practice. However, there are also significant risks of bias, threatening the validity of these studies. Research that re-use previously recorded health data has, by definition, a retrospective, observational (non-randomized) study design. This design is relatively weak for assessing causal effects, especially when compared to prospective, randomized, study designs. The main thread to validity of these research works is confounding, i.e., a lack of comparability between exposed and unexposed groups. The presence of confounding means that the exposed group is essentially different from the unexposed group at baseline. Had the exposed subjects actually been unexposed, their outcomes would be different from those in the actually unexposed group. For instance, it is conceivable that some of the people who underwent CT scanning in the study by Matthews and colleagues [27], described earlier in this paper, were tested because they had unexplained symptoms that later turned out to be caused by a cancer diagnosis. The authors have minimized the risk that this happened by excluding all patients that received a cancer diagnosis within one year after the CT scan - but we cannot exclude the possibility that there were other patients with a longer lead time. Similarly, neonatal care has probably improved during the years 2007-2011. Been and colleagues's interrupted time series design cannot distinguish the causal effects of such improvements from the effects of the smoking ban, which thus may be overestimated in the study [28].

A common method to address confounding is propensity scoring [73]. A propensity score is the estimated probability that an individual will be exposed to the intervention, conditional on observed baseline characteristics. A propensity score allows one to analyze observational data so that it mimics the characteristics of a randomized controlled trial. Propensity scores are typically constructed by training a probabilistic classifier (such as a logistic regression model) using the baseline characteristics as predictive features and exposure status as a class label. For instance, De Vries and colleagues [74] used generalized boosted regression to estimate the probability of receiving outpatient cardiac rehabilitation after hospitalization for a cardiac event or intervention, in a large insurance claims database (3.7 million people) from the Netherlands. Subsequently they applied Cox proportional hazards regression analysis with an inverse propensity score weighing to assess the effect of cardiac rehabilitation on survival. They found that rehabilitation reduced mortality risks by $35 \%$ in the first four years after the intervention.

Another way to address confounding is to select controls that are very similar to the exposed individuals - so-called 'matched controls'. Once a matched sample has been formed, the exposure effect can be estimated by directly comparing outcomes between exposed and unexposed subjects in the matched sample. Direct matching uses known confounders (e.g., age, sex, and diagnosis) to identify controls with exactly the same values for those variables. The number of matching variables and their categories must be kept small to prevent that no control would be found. An alternative is to match by propensity score, which typically allows inclusion of a larger number of confounders. However, if the propensity score is mis-specified, the covariates in the 
matched sample will be imbalanced, which can lead to a conditional bias. Therefore, a third, more recently developed approach, uses an evolutionary search algorithm to directly maximize covariate balance between exposed individuals and matched controls [75]. It does not depend on knowing or estimating a propensity score. Steventon and co-workers [76] used this 'genetic matching' technique in their evaluation of the effects of a telephonic alert system in patients with chronic obstructive pulmonary disease (COPD). The alert system used routine meteorological and communicable disease reports to identify times of increased health risks and then alerted the participants of the study. They found that this intervention increased hospital admission rates but reduced mortality.

When there is missing data (which will very often be the case when health data is re-used for research), this can pose significant challenges to the adjustment for confounding. For instance, in the administrative dataset used by De Vries and colleagues [74], there was no data available on cardiac function, a well-known confounder of survival for cardiac patients. It was probably assessed for most included patients but not included in the insurance claims that were re-used for the study. To address this problem, the authors used Lasso regression [77], a statistical feature selection method, to construct a set of proxies for cardiac function and other potential confounders from more than 900 variables that were available in the dataset (hospital diagnosis-treatment combinations, outpatient prescriptions, medical devices, occurrence of lab tests, GP visits, ICU days, and other services). The resulting set of 99 proxies included variables such as 'use of digitalis glycosides', a potent cardiovascular drug that is typically prescribed for patients with poor cardiac function.

This example illustrates that the breadth of routinely collected datasets can compensate for their modest fit with research purposes if we apply advanced machine learning and artificial intelligence techniques to discover the relevant features in data. Recently, researchers have started to use unsupervised deep learning methods to derive compact, feature-based, patient representations from
EHR data that are strongly predictive of health outcomes [49]. Such methods could further strengthen the existing array of propensity scoring and matching techniques.

\section{Analysis of Economic Benefit}

The development of EHRs and the use of their information for research purposes has been a recurrent theme in biomedical informatics, and it is a key and pivotal element for the development of what is currently known as precision medicine [78]. It must be noticed that in most of the cases when we refer to the use of EHR data, we mostly refer to data that is derived from an "ad-hoc" patient data repository or data warehouse, rather than the operational EHR itself [79].

Since the beginning of EHR implementation, there has been a clear interest in assessing and analyzing which were the economic impacts of introducing these technologies [80-84]. Most of the research carried out so far has been focused on the analyses in different areas such as:

\section{- Clinical care}

- Education

- Population and Global health

- Quality management and patient safety

However, and despite the increasing attention and the number of publications describing the secondary use of clinical data for research purposes, little research has been focused on the analysis of the economic aspects derived from this secondary (research) use of EHRs.

We conducted a series of literature searches in PubMed to assess to what extent this kind of analyses has been performed so far and what fields of biomedical research were the most commonly assessed from an economic perspective. These literature searches have been based on the use of different MeSH terms ("Biomedical Research", "Electronic Health Records" and "Economics"). At the time of writing this article (December 5, 2016), the Boolean search of these three terms together using the "AND" operator yielded a total of 90 documents, spanning from 2008 to 2016. In addition to that search, we also performed a second search using "Electronic Health Records/economics" [85] and "“Biomedical Research/economics"[MeSH Terms]" resulting in 7 articles.

Most of this research about the economic impacts of the secondary use of clinical information derived from EHRs for research purposes has been focused on a couple of areas, namely clinical trials and biobanks. The outcomes of these analyses are reflected in a still incipient literature which has been focused around two major dimensions:

- Quantitative vs. qualitative analyses. The major difference between these two approaches lies in the explicit analysis of the economic variable (quantitative analyses) or the use of other proxies and surrogate measurements that could be potentially linked with economic parameters such as time (qualitative analyses).

- Research phase. This dimension reflects the different stages (i.e. recruitment or execution) where the benefits of using data derived from EHRs may have an economic impact.

The European project EHR4CR is an excellent example of these research approaches. As a consequence of this work, A. Beresniak and colleagues published in January 2016 a research article that clearly shows the quantitative economic impact derived from the use of EHR-extracted data in three different clinical scenarios (protocol feasibility, patient selection, and study execution) [40]. On the other hand, Geisinger's MyCode project [86] is a recent example of some of the aforementioned application fields and dimensions (qualitative analyses on a biobank). Some other examples identified from the literature can be checked in Table 1 .

Although there is an increasing body of literature focusing on some of the "applied research" areas such as clinical trials, an important aspect that remains obscure in the literature is the impact of EHRs on fundamental research. To our knowledge, the actual economic impact of these systems applied to the so-called "fundamental/basic" research has not been yet assessed.

A recurrent topic for the development of what initially was known as personalized medicine and more recently has been called precision medicine was the use of data and 
Table 1 Examples of assessment of the economic impact derived from using EHRs for research.

\begin{tabular}{|l|l|l|}
\hline & Quantitative & Qualitative \\
\hline Subject Recruitment & $\begin{array}{l}\text { Massachusetts General Hospital } \\
\text { and Partners Healthcare Inc. Boston } \\
\text { estimated savings at \$7M per year [87]. }\end{array}$ & $\begin{array}{l}\text { Northwestern University was the \# 1 enrolling } \\
\text { U.S. sites (among 155 total sites) in a clinical } \\
\text { study of heart failure [89]. }\end{array}$ \\
\hline Study Execution & $\begin{array}{l}\text { At Vanderbilt University, the cost per } \\
\text { pharmacogenetic study was approx. }\end{array}$ & $\begin{array}{l}\text { Northwestern University Feinberg School of } \\
\text { Medicine needed to collect follow-up data on }\end{array}$ \\
& \$77,000 when using VESPA to link & heart failure patients admitted through the \\
& biobank with EHRs and enable cost & Northwestern Memorial Hospital. In four days, \\
& effective research as compared to & they electronically queried and refined the \\
& \$438,000 per study when not using & records for 40,953 ED admissions, 4,333 of \\
which met the criteria for the study [90].
\end{tabular}

information derived from EHRs in translational bioinformatics. The development of research projects and initiatives focused on the integration and combined analysis of genomic data and EHR-derived data have been of great interest during the last decade, e.g. GWAS or the eMERGE project. The economic impact associated with the award of those research grants based on the use and exploitation in research of EHR data is something that has not yet been considered.

\section{Conclusions}

This article has provided an overview of secondary use of patient data from a Big Data perspective. During our review, several common themes emerged. These include issues about data models, Big Data approaches to individual omics data, main advantages of these approaches, and concerns about data quality. We present them here in a special section before our review of recent and relevant examples of secondary use of patient data for purposes of clinical, genomic and health services research. We have also reviewed the economic impact of secondary use of clinical data. As we will witness in the next years the rise of very large collaborative research initiatives, such as the Precision Medicine Initiative (PMI, All of us Research Program) funded by the US government, we hope that this review may contribute to the identification of barriers, opportunities, and potential solutions. The PMI is a scientific endeavor that explicitly acknowledges the need to integrate environmental, behavioral, and genetic data with EHRs and patient(person-) generated data. The PMI Cohort Program aims at collecting data from one million or more participants, which will be made available to researchers, clinicians, and participants as a public repository. Precision medicine could both benefit and contribute immensely to the progress of the Learning Healthcare System paradigm. This model pursues an information ecosystem in which every clinical encounter becomes a source of data for clinical research, closing the loop between clinical care and biomedical research. This approach is a key step towards facilitating a truly personalized healthcare system.

\section{References}

1. Elliott JH, Grimshaw J, Altman R, Bero L, Goodman SN, Henry D, et al. Informatics: Make sense of health data. Nature 2015 Nov 5;527(7576):31-2.

2. Martin Sanchez F, Gray K, Bellazzi R, Lopez-Campos G. Exposome informatics: considerations for the design of future biomedical research information systems. J Am Med Inform Assoc 2014;21(3):386-90.

3. Mullins CD, Vandigo J, Zheng Z, Wicks P. Patient-centeredness in the design of clinical trials. Value Health 2014;17(4):471-5.

4. Tenenbaum JD, Avillach P, Benham-Hutchins M, Breitenstein MK, Crowgey EL, Hoffman MA, et al. An informatics research agenda to support precision medicine: seven key areas. J Am Med Inform Assoc 2016 Jul;23(4):791-5.

5. Geissbuhler A, Safran C, Buchan I, Bellazzi R, Labkoff S, Eilenberg K, et al. Trustworthy reuse of health data: A transnational perspective. Int J Med Inform 2013 Jan;82(1):1-9.

6. Curtis LH, Weiner MG, Boudreau DM, Cooper
WO, Daniel GW, Nair VP, et al. Design considerations, architecture, and use of the Mini-Sentinel distributed data system. Pharmacoepidemiol Drug Saf 2012 Jan;21 Suppl 1:23-31.

7. Overhage JM, Ryan PB, Reich CG, Hartzema AG, Stang PE. Validation of a common data model for active safety surveillance research. J Am Med Inform Assoc 2012;19(1):54-60.

8. Kohane IS, Churchill SE, Murphy SN. A translational engine at the national scale: informatics for integrating biology and the bedside. J Am Med Inform Assoc 2012;19(2):181-5.

9. Corley DA, Feigelson HS, Lieu TA, McGlynn EA. Building Data Infrastructure to Evaluate and Improve Quality: PCORnet. J Oncol Pract 2015 May;11(3):204-6.

10. 2016-11-15-PCORnet-Common-Data-Model-v3.1_Specification.pdf [Internet]. [cited 2016 Dec 12]. Available from: http://pcornet.org/ wp-content/uploads/2016/11/2016-11-15-PCORnet-Common-Data-Model-v3.1_Specification.pdf

11. Klann JG, Abend A, Raghavan VA, Mandl KD, Murphy SN. Data interchange using i2b2. J Am Med Inform Assoc 2016 Sep;23(5):909-15.

12. OHDSI - Observational Health Data Sciences and Informatics [Internet]. [cited 2016 Dec 12]. Available from: http://www.ohdsi.org/

13. Hripcsak G, Duke JD, Shah NH, Reich CG, Huser V, Schuemie MJ, et al. Observational Health Data Sciences and Informatics (OHDSI): Opportunities for Observational Researchers. Stud Health Technol Inform 2015;216:574-8.

14. Stephens ZD, Lee SY, Faghri F, Campbell RH, Zhai C, Efron MJ, Iyer R, Schatz MC, Sinha S Robinson GE, Big Data: Astronomical or Genomical? PLoS Biol 2015;13(7):e1002195.

15. O'Driscoll A, Daugelaite J, Sleator RD. ,Big data', Hadoop and cloud computing in genomics. J Biomed Inform 2013;46(5):774-81.

16. Tan A, Tripp B, Daley D. BRISK--research-oriented storage kit for biology-related data. Bioinformatics 2011;27(17):2422-5.

17. McConnell P, Dash RC, Chilukuri R, Pietrobon R, Johnson K, Annechiarico R, et al. The cancer translational research informatics platform. BMC Med Inform Decis Mak 2008;8:60.

18. Cerami E, Gao J, Dogrusoz U, Gross BE, Sumer SO, Aksoy BA, et al. The cBio cancer genomics portal: an open platform for exploring multidimensional cancer genomics data. Cancer Discov 2012;2(5):401-4.

19. Madhavan S, GusevY, Harris M, Tanenbaum DM, Gauba R, Bhuvaneshwar K, et al. G-DOC: a systems medicine platform for personalized oncology. Neoplasia 2011;13(9):771-83.

20. Shimokawa K, Mogushi K, Shoji S, Hiraishi A, Ido K, Mizushima H, et al. iCOD: an integrated clinical omics database based on the systems-pathology view of disease. BMC Genomics 2010;11 Suppl 4:S19.

21. Ohno-Machado L, Bafna V, Boxwala AA, Chapman BE, Chapman WW, Chaudhuri K, et al. iDASH: integrating data for analysis, anonymization, and sharing. J Am Med Inform Assoc 2012;19(2):196-201.

22. Szalma S, Koka V, Khasanova T, Perakslis ED. Effective knowledge management in translational 
medicine. J Transl Med 2010;8:68.

23. Canuel V, Rance B, Avillach P, Degoulet P, Burgun A. Translational research platforms integrating clinical and omics data: a review of publicly available solutions. Brief Bioinform 2015;16(2):280-90.

24. Kohane IS. HEALTH CARE POLICY. Ten things we have to do to achieve precision medicine. Science 2015;349(6243):37-8.

25. Gabetta M, Limongelli I, Rizzo E, Riva A, Segagni D, Bellazzi R. BigQ: a NoSQL based framework to handle genomic variants in i2b2. BMC Bioinformatics. 2015;16:415.Hersh WR. Adding value to the electronic health record through secondary use of data for quality assurance, research, and surveillance. Am J Manag Care 2007 Jun;13(6 Part 1):277-8.

26. Carey DJ, Fetterolf SN, Davis FD, Faucett WA, Kirchner HL, Mirshahi U, et al. The Geisinger MyCode community health initiative: an electronic health record-linked biobank for precision medicine research. Genet Med 2016;18(9):906-13.

27. Mathews JD, Forsythe AV, Brady Z, Butler MW, Goergen SK, Byrnes GB, et al. Cancer risk in 680,000 people exposed to computed tomography scans in childhood or adolescence: data linkage study of 11 million Australians. BMJ 2013;346:f2360

28. Been JV, Mackay DF, Millett C, Pell JP, van Schayck OC, Sheikh A. Impact of smoke-free legislation on perinatal and infant mortality: a national quasi-experimental study. Sci Rep 2015;5:13020..

29. Deeny SR, Steventon A. Making sense of the shadows: priorities for creating a learning healthcare system based on routinely collected data. BMJ Qual Saf 2015 Aug;24(8):505-15.

30. Bagley SC, Altman RB. Computing disease incidence, prevalence and comorbidity from electronic medical records. J Biomed Inform 2016 Oct; 63:108-111

31. Rusanov A, Weiskopf NG, Wang S, Weng C. Hidden in plain sight: bias towards sick patients when sampling patients with sufficient electronic health record data for research. BMC Med Inform Decis Mak 2014;14:51.

32. Terris DD, Litaker DG, Koroukian SM. Health state information derived from secondary databases is affected by multiple sources of bias. J Clin Epidemiol 2007;60(7):734-41.

33. Ancker JS, Kern LM, Edwards A, Nosal S, Stein DM, Hauser D, et al; HITEC Investigators. How is the electronic health record being used? Use of EHR data to assess physician-level variability in technology use. J Am Med Inform Assoc 2014;21(6):1001-8.

34. Safran C. Reuse of Clinical Data. Yearb Med Inform 2014 Aug 15;9(1):52-4.

35 . Hersh WR. Adding value to the electronic health record through secondary use of data for quality assurance, research, and surveillance. Am J Manag Care 2007 Jun;13(6 Part 1):277-8.

36. Jensen PB, Jensen LJ, Brunak S. Mining electronic health records: towards better research applications and clinical care. Nat Rev Genet 2012 May 2;13(6):395-405.

37. Electronic Health Records for Clinical Research - (EHR4CR) [Internet]. [cited 2016 Dec 12]. Available from: http://www.ehr4cr.eu/
38. De Moor G, Sundgren M, Kalra D, Schmidt A, Dugas M, Claerhout B, et al. Using electronic health records for clinical research: The case of the EHR4CR project. J Biomed Inform 2015 Feb;53:162-73.

39. Bruland P, McGilchrist M, Zapletal E, Acosta D, Proeve J, Askin S, et al. Common data elements for secondary use of electronic health record data for clinical trial execution and serious adverse event reporting. BMC Med Res Methodol [Internet]. 2016 Nov 22 [cited 2016 Dec 12];16. Available from: http://www.ncbi.nlm.nih.gov/pmc/articles/ PMC5118882/

40. Beresniak A, Schmidt A, Proeve J, Bolanos E, Patel $\mathrm{N}$, Ammour N, et al. Cost-benefit assessment of using electronic health records data for clinical research versus current practices: Contribution of the Electronic Health Records for Clinical Research (EHR4CR) European Project. Contemp Clin Trials 2016 Jan;46:85-91.

41. EMIF-European Medical Information Framework, http://www.emif.eu Last accessed 28 March 2017

42. Vaudano E, Vannieuwenhuyse B, Van Der Geyten S, van der Lei J, Visser PJ, Streffer J, et al. Boosting translational research on Alzheimer's disease in Europe: The Innovative Medicine Initiative AD research platform. Alzheimers Dement 2015 Sep;11(9):1121-2.

43. McMurry AJ, Murphy SN, MacFadden D, Weber G, Simons WW, Orechia J, et al. SHRINE: enabling nationally scalable multi-site disease studies. PLoS One 2013;8(3):e55811.

44. Richesson RL, Hammond WE, Nahm M, Wixted D, Simon GE, Robinson JG, et al. Electronic health records based phenotyping in next-generation clinical trials: a perspective from the NIH Health Care Systems Collaboratory. J Am Med Inform Assoc 2013 Dec;20(e2):e226-31.

45. Hripcsak G, Albers DJ. Next-generation phenotyping of electronic health records. J Am Med Inform Assoc 2013;20(1):117-21.

46. Rasmussen LV, Thompson WK, Pacheco JA, Kho AN, Carrell DS, Pathak J, et al. Design Patterns for the Development of Electronic Health Record-Driven Phenotype Extraction Algorithms. J Biomed Inform 2014 Oct; 0:280-6.

47. Liao KP, Cai T, Savova GK, Murphy SN, Karlson EW, Ananthakrishnan AN, et al. Development of phenotype algorithms using electronic medical records and incorporating natural language processing. BMJ [Internet]. 2015 Apr 24 [cited 2016 Dec 11];350. Available from: http://www.ncbi.nlm. nih.gov/pmc/articles/PMC4707569/

48. Yu S, Liao KP, Shaw SY, Gainer VS, Churchill SE, Szolovits P, et al. Toward high-throughput phenotyping: unbiased automated feature extraction and selection from knowledge sources. J Am Med Inform Assoc 2015 Sep;22(5):993-1000.

49. Miotto R, Li L, Kidd BA, Dudley JT. Deep Patient: An Unsupervised Representation to Predict the Future of Patients from the Electronic Health Records. Sci Rep [Internet] 2016 May 17 [cited 2016 Dec 11];6. Available from: http://www.ncbi. nlm.nih.gov/pmc/articles/PMC4869115/

50. Albers DJ, Elhadad N, Tabak E, Perotte A, Hripcsak G. Dynamical Phenotyping: Using Temporal Analysis of Clinically Collected Physiologic Data to Stratify Populations. PLoS ONE [Internet]
2014 Jun 16 [cited 2016 Dec 12];9(6). Available from: http://www.ncbi.nlm.nih.gov/pmc/articles/ PMC4059642/

51. Doshi-Velez F, Ge Y, Kohane I. Comorbidity Clusters in Autism Spectrum Disorders: An Electronic Health Record Time-Series Analysis. Pediatrics 2014 Jan;133(1):e54-63.

52. Hripcsak G, Albers DJ, Perotte A. Parameterizing time in electronic health record studies. J Am Med Inform Assoc 2015 Jul;22(4):794-804.

53. Hripcsak G, Mirhaji P, Low AF, Malin BA. Preserving temporal relations in clinical data while maintaining privacy. J Am Med Inform Assoc 2016 Nov 1;23(6):1040-5.

54. Gabetta M, Malovini A, Bucalo M, Zini E, Tibollo V, Priori SG, et al. Beyond Cohort Selection: An Analytics-Enabled i2b2. Stud Health Technol Inform 2016;228:572-6.

55. Hu Z, Jin B, Shin AY, Zhu C, Zhao Y, Hao S, et al. Real-Time Web-Based Assessment of Total Population Risk of Future Emergency Department Utilization: Statewide Prospective Active Case Finding Study. Int J Med Res 2015 Jan 13;4(1):e2.

56. Segagni D, Sacchi L, Dagliati A, Tibollo V, Leporati P, De Cata P, et al. Improving Clinical Decisions on T2DM Patients Integrating Clinical, Administrative and Environmental Data. Stud Health Technol Inform 2015;216:682-6.

57. van Teeffelen SR, Douglas CM, van El CG, Weinreich SS, Henneman L, Radstake M, et al. Mothers' Views on Longer Storage of Neonatal Dried Blood Spots for Specific Secondary Uses. Public Health Genomics 2016;19(1):25-33.

58. Sen A, Heredia N, Senut MC, Land S, Hollocher $\mathrm{K}, \mathrm{Lu} \mathrm{X}$, et al. Multigenerational epigenetic inheritance in humans: DNA methylation changes associated with maternal exposure to lead can be transmitted to the grandchildren. Sci Rep 2015;5:14466.

59. Poon AF, Gustafson R, Daly P, Zerr L, Demlow SE, Wong J, et al. Near real-time monitoring of HIV transmission hotspots from routine HIV genotyping: an implementation case study. Lancet HIV 2016;3(5):e231-8.

60. Ritchie SC, Wurtz P, Nath AP, Abraham G, Havulinna AS, Fearnley LG, et al. The Biomarker GlycA Is Associated with Chronic Inflammation and Predicts Long-Term Risk of Severe Infection. Cell Syst 2015;1(4):293-301.

61. Kho AN, Pacheco JA, Peissig PL, Rasmussen L, Newton KM, Weston N, et al. Electronic medical records for genetic research: results of the eMERGE consortium. Sci Transl Med 2011;3(79):79re1.

62. Krishnamoorthy P, Gupta D, Chatterjee S, Huston J, Ryan JJ. A review of the role of electronic health record in genomic research. J Cardiovasc Transl Res 2014;7(8):692-700.

63. Gottesman O, Kuivaniemi H, Tromp G, Faucett WA, Li R, Manolio TA, et al. The Electronic Medical Records and Genomics (eMERGE) Network: past, present, and future. Genet Med 2013;15(10):761-71.

64. Verma SS, Cooke Bailey JN, Lucas A, Bradford Y, Linneman JG, Hauser MA, et al. Epistatic Gene-Based Interaction Analyses for Glaucoma in eMERGE and NEIGHBOR Consortium. PLoS Genet 2016;12(9):e1006186. 
65. Namjou B, Marsolo K, Lingren T, Ritchie MD, Verma SS, Cobb BL, et al. A GWAS Study on Liver Function Test Using eMERGE Network Participants. PloS One 2015;10(9):e0138677.

66. Almoguera B, Vazquez L, Mentch F, Connolly J, Pacheco JA, Sundaresan AS, et al. Identification of Four Novel Loci in Asthma in European and African American Populations. Am J Respir Crit Care Med 2017 Feb 15;195(4):456-63.

67. Dumitrescu L, Goodloe R, Bradford Y, Farber-Eger E, Boston J, Crawford DC. The effects of electronic medical record phenotyping details on genetic association studies: HDL-C as a case study. BioData Min 2015;8:15.

68. Crawford DC, Goodloe R, Farber-Eger E, Boston J, Pendergrass SA, Haines JL, et al. Leveraging Epidemiologic and Clinical Collections for Genomic Studies of Complex Traits. Hum Hered 2015;79(3-4):137-46.

69. Bush WS, Crosslin DR, Owusu-Obeng A, Wallace J, Almoguera B, Basford MA, et al. Genetic variation among 82 pharmacogenes: The PGRNseq data from the eMERGE network. Clin Pharmacol Ther 2016;100(2):160-9.

70. Denny JC, Bastarache L, Roden DM. Phenome-Wide Association Studies as a Tool to Advance Precision Medicine. Annu Rev Genomics Hum Genet 2016;17:353-73.

71. Bush WS, Oetjens MT, Crawford DC. Unravelling the human genome-phenome relationship using phenome-wide association studies. Nat Rev Genet 2016;17(3):129-45.

72. Gottlieb L, Tobey R, Cantor J, Hessler D, Adler NE. Integrating Social And Medical Data To Improve Population Health: Opportunities And Barriers. Health Aff 2016;35(11):2116-23.

73. Austin PC. An Introduction to propensity score methods for reducing the effects of confounding in observational studies. Multivariate Behav Res 2011;46:399-424.

74. De Vries H, Kemps HM, van Engen-Verheul MM, Kraaijenhagen RA, Peek N. Cardiac reha- bilitation and survival in a large representative community cohort of Dutch patients. Eur Heart J 2015;36(24):1519-28.

75. Sekhon JS, Grieve RD. A matching method for improving covariate balance in cost-effectiveness analyses. Health Econ 2012;21(6):695-714.

76. Steventon A, Bardsley M, Mays N. Effect of a telephonic alert system (Healthy outlook) for patients with chronic obstructive pulmonary disease: a cohort study with matched controls. J Public Health 2015;37(2):313-21.

77. Tibshirani R. Regression shrinkage and selection via the Lasso. J Royal Stat Soc B 1996;58:267-88.

78. Ritchie MD, de Andrade M, Kuivaniemi H. The foundation of precision medicine: integration of electronic health records with genomics through basic, clinical, and translational research. Front Genet 2015;6:104.

79. Hersh WR, Weiner MG, Embi PJ, Logan JR, Payne PRO, Bernstam EV, et al. Caveats for the Use of Operational Electronic Health Record Data in Comparative Effectiveness Research. Med Care 2013;51(8):S30-S7.

80. Bassi J, Lau F. Measuring value for money: a scoping review on economic evaluation of health information systems. J Am Med Inform Assoc 2013;20(4):792-801.

81. Menachemi N, Brooks RG. Reviewing the benefits and costs of electronic health records and associated patient safety technologies. J Med Syst 2006;30(3):159-68.

82. Park H, Lee SI, Hwang H, Kim Y, Heo EY, Kim JW, et al. Can a health information exchange save healthcare costs? Evidence from a pilot program in South Korea. Int J Med Inform 2015;84(9):658-66.

83. Pisano F, Lorenzoni G, Sabato SS, Soriani N Narraci O, Accogli M, et al. Networking and data sharing reduces hospitalization cost of heart failure: the experience of GISC study. J Eval Clin Pract 2015;21(1):103-8.

84. Boonstra A, Versluis A, Vos JF. Implementing electronic health records in hospitals: a system- atic literature review. BMC Health Serv Res 2014;14:370.

85. Davis AP, Wiegers TC, Rosenstein MC, Mattingly CJ. MEDIC: a practical disease vocabulary used at the Comparative Toxicogenomics Database. Database (Oxford) 2012;2012:bar065.

86. Casey JA, Schwartz BS, Stewart WF, Adler NE. Using Electronic Health Records for Population Health Research: A Review of Methods and Applications. Annu Rev Public Health 2016;37:61-81.

87. Nalichowski R, Keogh D, Chueh HC, Murphy SN. Calculating the benefits of a Research Patient Data Repository. AMIA Annu Symp Proc 2006:1044.

88. Bowton E, Field JR, Wang S, Schildcrout JS, Van Driest SL, Delaney JT, et al. Biobanks and electronic medical records: enabling cost-effective research. Sci Transl Med 2014;6(234):234cm3

89. NUCATS drives top recruiting in NIH-funded heart failure trials and „big data“ analysis in novel clinical program [cited 2016 Dec 12]. Available at: http://nucats.northwestern.edu/about/successstories/drives-top- recruiting-nih-funded-heartfailure-trials-big-data-analysis-novel-clinicalprogram

90. RAPID evaluates transplant clinic performance \& patient outcomes in real-time [cited 2016 Dec 12]. Available at: http://nucats.northwestern.edu/about/ success-stories/rapid-evaluates-transplant-clinicperformance-patient-outcomes-real-time

\section{Correspondence to:}

Fernando J. Martin-Sanchez, Ph.D. FACHI, FACMI

Weill Cornell Medicine

Department of Healthcare Policy and Research

Division of Health Informatics

425 East 61 st Street

New York, NY 10065, USA

Tel: + (1) 6469629438

E-mail: fem2008@med.cornell.edu 